\title{
Relatos de viaje a la URSS y giro emocional: César Vallejo y Rafael Alberti
}

Resumen: Con el giro emocional en las ciencias sociales y las humanidades, los relatos de viaje son terreno fértil para estudiar las relaciones de los intelectuales con las sociedades que visitan y la fricción emocional que surge del contacto. En el caso de la Unión Soviética, la radicalidad del experimento político atrajo a intelectuales de diversas posiciones ideológicas (la mayoría de las veces, simpatizantes con el proyecto ideológico). Dos de ellos fueron los escritores César Vallejo y Rafael Alberti; su labor primordialmente poética y su militancia política confluyen en los textos que ambos elaboraron a partir de su experiencia en la URSS. En dichos textos, entre diversos aspectos del cosmos socialista, se trata el asunto de las emociones del nuevo ciudadano soviético. Este artículo desarrolla reflexiones y propone algunas líneas de análisis sobre esta cuestión.

Palabras clave: emociones, viaje a la URSS, ciudadano soviético, estalinismo, literatura iberoamericana

\section{Los viajes a la URSS y el giro emocional}

César Vallejo y Rafael Alberti hicieron sus primeros viajes a la Unión Soviética (en el caso de Vallejo, los únicos) durante la consolidación del estalinismo. Al ser ambos autores punta de lanza poética de la comunidad intelectual hispanoamericana, los viajes de Vallejo y Alberti repercuten en la historia literaria de sus propias tradiciones; asimismo, pertenecen a un conjunto más amplio de testimonios de viajeros a la Unión Soviética - un "nuevo mundo" político que aparece a partir de la Revolución de 1917 (con la Rusia bolchevique) y que se conforma con la fundación de la Unión de Repúblicas Socialistas Soviéticas en 1922-. Sus proyectos respectivos, en tanto escritores, comprenden una producción textual en prosa que analiza y sintetiza los ejercicios intelectuales originados por sus desplazamientos,

Este artículo se desprende de mi investigación doctoral sobre la literatura de viaje a la URSS de escritores iberoamericanos, que desarrollo en la Universidad de Potsdam, con una beca del Deutscher Akademischer Austauschdienst para estudios de doctorado y bajo la supervisión del Prof. Dr. Ottmar Ette.

Rodrigo García Bonillas, Universität Potsdam

Ә Open Access. (C) 2021 Rodrigo García Bonillas, published by De Gruyter. (c) BY BY This work is licensed under the Creative Commons Attribution 4.0 International License. https://doi.org/10.1515/9783110727555-005 
con el fin de observar nuevas organizaciones políticas y sociales, así como nuevos hábitos de socializar y experimentar emociones entre los ciudadanos soviéticos. Buena parte de esa producción textual, como en el caso de los dos escritores que aquí se considera, se publicó primero en la prensa periódica y tuvo gran difusión. ${ }^{1}$

Con el giro emocional de las ciencias sociales y las humanidades -“emotional turn", "turn to the emotions", "turn to the affect” (Leys 2011: 434-435; Weyher 2012: 342)-, cabe preguntarse por las implicaciones de lo emocional en la tipología textual del viaje a la Unión Soviética y, en general, de los "peregrinajes políticos" (Hollander 1981: vii), en tanto desplazamientos humanos con un evento histórico violento -la Revolución rusa- como punto de fuga, sobre todo en el horizonte de las dos primeras décadas de la URSS. En este sentido y en relación con la producción cultural de origen proletario, apunta Sabine Hake:

Much of the power of emotions in social movements derives from their formative, if not transformative functions - namely, to provide identities, create communities, and sustain identifications and commitments. For these reasons, calls for revolutions, whether democratic, socialist, or nationalist, tend to be written in the language of emotions. (Hake 2017: 1)

La indagación sobre las emociones en esta tradición textual concierne a los mecanismos que los viajeros emplearon en sus escritos para configurar las emociones desprendidas del desplazamiento geográfico. Podría pensarse que el viaje de tinte político origina la exposición ante sensibilidades diferentes y una inestabilidad de las propias emociones; sin embargo, si militan en las izquierdas y son prosoviéticos, los escritores tienden más bien a encontrar en el viaje lo que ya traían desde casa: una simpatía a ultranza con sus hermanos en la ideología (en el sentido de Hake y Hollander) y, por lo tanto, signos de ese entendimiento sonrisas, abrazos, gestos de generosidad y alegría, cantos, lágrimas, conversaciones vivaces, etcétera-.

Desde el punto de vista de las emociones, ¿qué se puede estudiar en estos tipos de textos? ¿Con qué fines? En el caso específico de Alberti, es posible observar los relatos de su encuentro con el pueblo soviético desde la perspectiva de lo emocional: descripciones de los momentos de pathos; tonos amistosos o beligerantes que el autor toma frente a diferentes actores políticos y poéticos;

1 En la tesis doctoral que desarrollo actualmente (cf. nota al pie de la página 85) estudio un corpus que incluye nueve autores iberoamericanos de diversos tiempos y espacios que incursionaron en este género textual: Fernando de los Ríos, Josep Pla, los propios Vallejo y Alberti, María Teresa León, José Revueltas, Luis Cardoza y Aragón, Graciliano Ramos y Gabriel García Márquez (en esta enumeración sigo el orden cronológico del primer viaje a la URSS de cada uno de estos autores). A partir de este corpus presento la lectura de un panorama que abarca temporalmente desde el ascenso bolchevique en Rusia (De los Ríos) hasta la Revolución cubana (García Márquez). En dicho trabajo doctoral expongo las reflexiones teóricas que conciernen a este género. 
rasgos gráficos (o prosódicos), sintácticos y léxicos de su prosa, así como figuras retóricas; y sus propias observaciones sobre la realidad soviética en el ámbito de los sentimientos o las emociones (en un sentido metarreferencial). En el caso de Vallejo, además de todos estos elementos existe la teorización sobre las emociones y los afectos en la URSS, lo que ofrece un examen y un concepto de las transformaciones originadas por la Revolución bolchevique en el ámbito de lo emocional y lo afectivo. El pensamiento alrededor de esta cuestión es más intenso en los reportajes vallejianos y tiene mayores intenciones suasorias; sin embargo, por esos mismos rasgos la condición de sus aseveraciones es hoy menos verosímil.

Queda aún la pregunta sobre los fines de este ejercicio. Que siga pendiente: vayamos ahora a los casos.

\section{Nuevo mundo, nuevas emociones}

La instauración de la Unión Soviética como entidad política, económica y social, aislada del resto del mundo (capitalista), hereda una serie de tópicos sobre el modo de sentir revolucionario -el compañerismo entre "camaradas"- y adopta una serie de dogmas políticos que se desprenden de la implantación del marxismo-leninismo como única teoría político-filosófica. Ambas inserciones se enfrentan con las peculiaridades de los pueblos que las adquirieron, a veces colisionando. Nuevas maneras de emocionarse, según los testigos viajeros, surgen con rapidez. Muy pronto, hacia mediados de los años veinte, los viajeros a la URSS ya perciben o creen percibir una nueva configuración ética de los ciudadanos soviéticos. ${ }^{2}$ El desplazamiento de estos viajeros, motivado por la búsqueda de la vivencia del experimento soviético, ya sea por razones heurísticas, ya por ideológicas, ya por ambas, les permite observar y analizar la realidad presentada en carne y hueso. Al calor de eventos históricos decisivos, muchos de estos viajeros configurarán por escrito su experiencia con el propósito de la divulgación en los países de origen o las comunidades político-intelectuales que los reciben -con mayor o menor éxito en el circuito-.

2 Por ejemplo, Vallejo señala este cambio una década después de la revolución: "Social y económicamente, todos son iguales. El individualismo y sus apetitos derivados tienen un freno dentro de un nuevo equilibrio colectivo y dentro de un nuevo orden jurídico y moral. El trabajo es obligatorio. No hay tiempo para el ocio ni gusto por los refinamientos. A la vanidad ha sucedido el orgullo, en aceptación colectiva de la palabra” (Vallejo 2002: 67; volveré a esta cita). 
Por una parte, César Vallejo viaja por primera vez a la URSS en 1928 desde París, vía Berlín; vuelve en 1929 con Georgette Vallejo -su pareja- y, finalmente, en 1931, por invitación de la VOKS (Sociedad Pansoviética de Relaciones Culturales con el Extranjero). Una vasta producción textual -ensayística y periodística- deriva de esos periplos: el libro de reportajes Rusia en 1931. Reflexiones al pie del Kremlin (1931), con su secuela, Rusia ante el Segundo Plan Quinquenal (1965), y el libro de ideas El arte y la revolución (1973). La mayor parte de este material se publicó póstumamente (cf. Priego en: Vallejo 2002: CVII-CIX). La gran excepción fue Rusia en 1931, que gozó de gran éxito en el momento de su aparición (cf. Priego en: Vallejo 2002: 17). ${ }^{3}$ En estas obras, Vallejo estudia exhaustivamente la Unión Soviética en sus diversas facetas y a través de una retórica militante, resuelta y tenaz.

Por otra, las transformaciones éticas de Alberti -el segundo viajero que aquí se aborda- hacia finales de la década de los veinte y principios de los treinta forman una coyuntura entre la vida emocional del poeta (con sus repercusiones líricas) y las tensiones internacionales que preparan la Segunda Guerra Mundial, con la guerra civil española como preludio. Como señala Miguel Ángel García, al paso al “compromiso político" lo precede una “crisis":

Lo que ahora reprueba [Pedro Salinas] es que Alberti ya se encuentra incluso más allá del surrealismo, o del nuevo romanticismo y de Bécquer [...], de la estética irracionalista con la cual ha canalizado en Sobre los ángeles, tras su paso por el neopopularismo y el fervor gongorino, una crisis personal, poética y social [...] que puede considerarse la antesala de su compromiso político. (García 2017: 51)

Esa crisis se supera y es sucedida por un sonado giro ideológico a inicios de la década de los treinta, en una situación vital donde Alberti establece con la escritora María Teresa León un compromiso sentimental e ideológico. León y Alberti serán compañeros en viaje a la Unión Soviética y juntos participarán en el proceso de socialización con los ciudadanos soviéticos, ya sea en mítines en el Teatro Bolshói o en el propio Kremlin; asimismo, algunos de sus textos poéticos y memorialísticos tendrán correspondencias entre sí y se referirán a experiencias compartidas, como se puede observar en varios pasajes que pertenecen tanto a Memoria de la melancolía (1970) de León, como a La arboleda perdida (19591987) de Alberti. En ese sentido, los primeros años de la década de los treinta son una época de una nueva educación sentimental para Alberti en sentido poético, social y político. Por el espíritu de la época, esa educación sentimental propia

3 Para una breve relación de la historia textual de estas obras vallejianas, véase la nota editorial de Manuel Miguel de Priego en: Vallejo 2002: CVII-CIX. 
será proyectada a las amplias esferas de lo político y lo histórico. Además de las memorias, de Alberti nos quedan dos series periodísticas (una de ellas publicada en Prosas encontradas), que el hispanista Robert Marrast rescató en 1969 y 1986, respectivamente: la primera es de 1932 a 1933, y la segunda de 1934; y ambas se publicaron en el periódico efímero Luz (cf. Alberti 1969: 335; Alberti 1986: 357). Finalmente, algunos recuerdos del viaje de 1937 a la URSS, en plena Guerra Civil, se pueden encontrar en Memoria de la melancolía.

El precedente del viaje de la pareja a la URSS es la estancia de varios meses en algunas capitales de Europa, con el fin de investigar los movimientos teatrales de vanguardia (cf. García 2017: 54-55). La elección del trato con la gente de izquierda tiene su extremo en la Alemania del ascenso del fascismo, donde Alberti solo puede trabar relación con un sector político: "Ando con algunos comunistas, la única gente tratable y decente de Alemania” (Alberti en: Cabrol 2004: 122). En Rusia puede, por sus filiaciones ideológicas, conocer a un espectro de personajes que van desde obreros y trabajadores de un crematorio hasta Iósif Stalin (en 1937), pasando, por supuesto, por sus colegas, los artistas e intelectuales de la URSS. La simpatía unánime que le despiertan todos estos individuos modela la conformación del ethos de sus interlocutores, y, por otro lado, a partir de esta época Alberti repudiará en sus textos, incluso en los poéticos, a los individuos de ideología contraria. Así, por ejemplo, sobre las declaraciones prosoviéticas de Gide, señala que fueron "recibidas con mordeduras y ladridos de la otra banda" (Alberti 1969: 346).

Con la excepción de Stalin, Vallejo también conocerá a personajes afines a los que conoció Alberti: obreros, intelectuales, políticos y, en general, individuos de a pie que representan distintos tipos de la sociedad soviética. Las intenciones heurísticas de Vallejo, no obstante, lo llevarán a encuentros que se presentan de manera más profunda e intensa en sus reportajes. El tratamiento intenta evitar "la crítica sentimental y subjetiva” (Vallejo 2002: 6) con respecto a la Unión Soviética, pero dedica varios pasajes a analizar las emociones y las pasiones en el nuevo sistema político. Asimismo, en algunos pasajes clave la voz del reportero se emociona y así contradice expresamente el pretendido "carácter de imparcialidad" (Vallejo 2002: 50) de su reportaje. ${ }^{4}$ Según Sara Ahmed, un tópico en cuanto a las

4 Hay varios ejemplos del paso a lo subjetivo y a las emociones, por lo general marcados con signos de admiración. Menciono aquí dos en particular: ante un "haraposo" que recoge "migajas y desperdicios de una mesa”, Vallejo le señala a su acompañante (una joven komsomolka, es decir, miembro del Komsomol o Unión Comunista de la Juventud): “iEspantoso!”, y concluye, “[l] as miradas del hambriento sobre el pastel son febriles y casi rabiosas. Nunca he visto ojos tan extraños en mi vida. Hay en la cara de este pobre una avidez agresiva, furiosa, demoníaca. A veces tengo la impresión de que va a saltar sobre nosotros y nos va a arrancar de un zarpazo un trozo de nuestras carnes. Se ve que tiene cólera. Se ve que nos odia con todas sus entrañas de 
emociones es el asociar "pasión y pasividad": "To be emotional is to have one’s judgement affected: it is to be reactive rather than active, dependent rather than autonomous" (Ahmed 2014: 3). Vallejo quiere evitar lo "sentimental" y lo "subjetivo" porque ambas categorías van en contra de la nueva concepción emocional de la sociedad sana y vigorosa, para cuya constatación ha organizado un viaje.

\section{Emoción y relaciones humanas en la URSS}

En el cruce de la frontera de la URSS se exalta, en primer lugar, el ánimo, a pesar de las condiciones climáticas adversas: “¿Qué es este impulso, este nuevo latido de la sangre, este rápido vuelco que nos hace saltar de los asientos y descorrer los cristales helados?” (Alberti 1969: 336). La penetración tiene efectos físicos inéditos que corresponden a nuevas maneras de emocionarse según la clase social o la ideología política. En ese fragmento de los relatos de Alberti se combina la expectativa del viaje (en tanto turismo político) con la estimulación del nuevo país. El episodio, según una típica fórmula, se representa de manera intensa, casi hiperbólica, y totalmente simpática con los ciudadanos soviéticos: una pregunta retórica, una división en tres cláusulas y la oposición entre lo sanguíneo y lo helado -en la cual lo primero se sobrepone a lo segundo-. La cara oscura del régimen bolchevique que hoy se impone vuelve fallido el sentimentalismo de la escena y lo convierte en un kitsch político, pero para el lector madrileño de la época convulsa las condiciones eran distintas. La emoción del viajero en la URSS debió de ser abono para el círculo de simpatías entre las izquierdas en relación con el proyecto soviético, que empezaría a hacer aguas a fines de la década de los treinta por diferentes razones, como el papel de la URSS durante la guerra civil española; algunos relatos de viaje a la URSS como los de Panait Istrati (Vers l'autre flamme, 1929) o André Gide (Retour de l'U.R.S.S., 1936); o el Pacto Ribbentrop-Mólotov de 1939.

\footnotetext{
hambriento. Inspira miedo, respeto y una misericordia infinita. ¡El apetito es, sin duda, una cosa horrorosa!" (Vallejo 2002: 126). Momentos similares de pathos frente a los representantes de las clases "antirrevolucionarias" (gente sin ocupación, burgueses, antiguos aristócratas, cristianos, etcétera) ocurren en varios pasajes de los reportajes de Vallejo y el autor reacciona, por lo general, con efusivo desagrado o consternación. Frente a un grupo de campesinos creyentes, Vallejo indica: "[...] lo que hay de cierto es que sus caras de hambrientos, su desnudez, sus miradas llenas de angustiosa incertidumbre, su canto, todo en ellos está henchido de tragedia. Sus voces y sus ojos expresan un terror misterioso, vago, aunque real y viviente. [...] Toda la vida, todo el dolor y todos los dramas y conflictos de su ser profundo se agitan ahora en sus miradas y en sus voces. $\mathrm{Y}$ no hay cosa más insondable que el canto y la mirada de los hombres” (Vallejo 2002: 135).
} 
El proceso de simpatía tiene en las crónicas de Alberti un signo en la reiteración de las sonrisas de las personas con las que se encuentra. A falta de comunicación directa, el viajero en la Unión Soviética que ignora el ruso o alguna de las lenguas vernáculas depende de las palabras de un tercero para la comunicación, pero siempre recurre a los gestos esenciales que indican una emoción para entender los afectos cotidianos de ese mundo. Por la simpatía, es recurrente la aparición de sonrisas y gestos de afecto o buen humor, que hablan, sobre todo, de una sociedad sana y expresiva -si bien la historia contaría otras cosas al respecto sobre esa misma sociedad y sus crímenes, como se expone, por ejemplo, en Le Livre noir du communisme (cf. Courtois et al. 1997), la obra crucial sobre el tema-. ${ }^{5}$ En 1934 el autor visita Rusia durante el verano (a diferencia del viaje anterior, que sucedió durante el invierno) y señala: "Los ojos de la gente van contentos y la expresión de todos los rostros es de seguridad y alegría” (Alberti 1986: 367). Más adelante observa a los obreros, "constructores del socialismo”, que pasan "sonriendo a su obra”, “diminutos, orgullosos”, y luego la atmósfera general, con un nosotros que lo incluye en la obra de construcción: "Estamos alegres, estamos fuertes", mientras que "el camarada Stalin puede estar satisfecho” (Alberti 1986: 368). Según un tópico de la opinión prosoviética, el pueblo estima a Stalin y por lo tanto la satisfacción del líder es la satisfacción general.

Lo sano es otro tópico de los viajeros simpatizantes. ${ }^{6}$ La experiencia de la Unión Soviética implica, tanto para Vallejo como para Alberti, entrar en contacto con una sociedad modelo, donde el eje de las emociones se ha transformado, desterrando los hábitos mezquinos, propios de la burguesía o del Antiguo Régimen. ${ }^{7}$ Vallejo observa en la pareja proletaria un nuevo tipo de amor "no clasista", distinto del "amor" por interés de las sociedades burguesas. En ese sentido, el destierro de las supuestas formas de amor basadas en una pertenencia de clase permite que se establezcan relaciones de afectividad "pura":

En Rusia, el amor ha dejado de ser clasista, desde el momento en que han desaparecido las clases sociales. Social y económicamente, todos son iguales. [...] A la vanidad ha sucedido el orgullo, en aceptación colectiva de la palabra. El hombre y la mujer, por consiguiente, están liberados de toda preocupación o perspectiva económica y social para elegir a la compañera

5 Gómez L-Quiñones y Winter exponen el asunto de los crímenes soviéticos y Le Livre noir du communisme en la introducción a Cruzar la línea roja. Hacia una arqueología del imaginario comunista ibérico (1920-2017) (cf. Gómez L-Quiñones / Winter 2017: 9-43).

6 Desde la perspectiva de Vallejo, lo sano es la condición para que los ciudadanos puedan participar de una vida emocional positiva y políticamente constructiva.

7 Vallejo señala sobre la ciudad ("socialista”) del futuro: "cuando la sociedad cesa de ser una jauría de groseros individualismos, un lupanar de instintos bestiales $-\mathrm{y}$ menos que bestiales, viciosos-" (Vallejo 2002: 14). 
o al compañero. El punto de partida y de inspiración del amor radica por entero en otra parte: en el mundo afectivo. Dentro de este mundo, la libertad de elección sentimental es absoluta e inalienable. (Vallejo 2002: 67)

En uno de sus reportajes, Vallejo observa con atención a una pareja formada por una periodista de Pravda y un albañil: en ellos descubre cierto ánimo cándido y sano: "Ella le habla y obra espontáneamente. Él se muestra un tanto paternal. Ambos son alegres, ágiles, infantiles. Ríen y juegan mientras se lavan y visten para ir al trabajo" (Vallejo 2002: 68). La revolución emocional configura también nuevos modos de relacionarse socialmente y de ejercer la sexualidad. Con independencia de las modificaciones posteriores, la Unión Soviética implementó políticas en el ámbito de las relaciones de pareja: "El marido y la mujer soviéticos son, ante todo, buenos amigos. El amor conyugal en Rusia es más amistad que pasión, más fraternidad que atracción sexual” (Vallejo 2002: 76). Conforme evidencia el pasaje citado, esta pareja tiene muy pocas demostraciones de afecto: más que cónyuges, son camaradas.

Debido a la moralidad característica de la época, las variaciones en el ámbito sexual eran entonces motivo de escándalo fuera de la URSS. La unión libre (o "amor libre"), el divorcio ${ }^{8}$ y el aborto eran derechos inéditos en la historia moderna que la Unión Soviética instituía; además eran una realidad con efecto sobre ciertas poblaciones vulnerables, como las madres solteras o los hijos fuera del matrimonio. En ese ámbito, Vallejo presenta algunas reflexiones sobre el deseo, que expone a manera de diálogo con Ana Virof, una trabajadora de la Casa del Campesino que tiene experiencia en la cuestión de la maternidad. Desde el punto de vista de Virof, con el que Vallejo coincide, el matrimonio, en tanto institución burguesa -y, en consecuencia, "antirrevolucionari[a]"-, supuestamente "tiende a desaparecer" en la URSS de los años veinte (Vallejo 2002: 139, 141). Si bien esto no ocurrió, sí es cierto que la cuestión era novedosa en Occidente y por lo tanto diversos viajeros se ocuparon de ella. En principio, Vallejo acusa -a través de la entrevista con Virof- una distorsión de los aspectos sexuales y familiares de la URSS con respecto a la imagen de la misma en el extranjero: en vez de un desenfreno o un "libertinaje", encuentra allí una "monogamia rigurosa y austera", capaz de una "mayor moralidad" (Vallejo 2002: 137); la "pesadilla del deseo" de las sociedades burguesas ya no existe y el "clima social" ya no es corrupto, pues ahí el "aire se purifica" (Vallejo 2002: 138).

8 En cuanto al divorcio, Vallejo se entera de que la petición de solo uno de los cónyuges es suficiente para anular el matrimonio. 
Vallejo dedica en particular dos capítulos de su obra Rusia en 1931 a exponer las nuevas sentimentalidades soviéticas: el primero es el reportaje del obrero casado con la periodista del Pravda; el segundo es la conversación con Ana Virof, quien le explica las peculiaridades de la flamante familia soviética. Hay, por supuesto, muchos pasajes donde se refiere al asunto de las emociones con un enfoque negativo o positivo, dependiendo de la clase a la que se pertenece, y con una visión binaria, donde lo decadente se opone a lo progresivo. Lo más relevante es la nueva sentimentalidad que una cultura, que basa su conformación en el entramado de la filosofía política marxista y que se concibe como la encarnación de la dictadura del proletariado, construye no solo en la relación entre los hombres, sino con respecto a las máquinas y el trabajo en general. Así, Vallejo cita a [Fiódor] Gladkov ("La nostalgia de las máquinas es más fuerte que la nostalgia del amor") y desarrolla la tesis del trabajo (y la producción económica) como sustento de la vida social (cf. Vallejo 2002: 143, 152). De esa manera, la cuestión emocional se subordina al núcleo "verdadero" de la actividad humana y las nuevas sentimentalidades obedecen, por lo tanto, a este motor primero.

\section{Cierre}

¿Cuál es el fin de estudiar las emociones en la literatura de viaje a la URSS? Hasta ahora se ha hablado de las emociones en general, si bien tanto en Alberti como en Vallejo -sobre todo en Vallejo- hay una clasificación que enaltece algunas y discrimina otras, dependiendo de su temporalidad histórica (prerrevolucionaria o socialista) y de su clase social (burguesa o proletaria). Dos cuestiones me parecen sintomáticas de esta axiología: las relaciones amorosas y las relaciones con la muerte. En cuanto a lo primero, para Vallejo el amor fuera de la URSS -o lo que según él "llamamos amor" (Vallejo 2002: 65) - es "una simple simpatía hija directa de un interés económico o de cualquiera otra especie, pero que nada tiene que ver con el mundo afectivo" (Vallejo 2002: 65). Vallejo concluye que esto sucede por el "individualismo desenfrenado de las gentes" (Vallejo 2002: 66) y también que "el amor -si así puede llamarse entre nosotros ese apetito- es clasista" (Vallejo 2002: 66. Las cursivas son mías), mientras que "en Rusia, el amor ha dejado de ser clasista": "El punto de partida y de inspiración del amor radica por entero en otra parte: en el mundo afectivo. [...] Cuando un hombre está unido a una mujer, se supone que lo está por el amor, puesto que no hay otra cosa o interés que pueda unirlos" (Vallejo 2002: 67). En cuanto a la cultura funeraria, Alberti visita al final de su primer viaje a la URSS un crematorio, donde puede observar la relación de los ciudadanos soviéticos con la muerte: 
Fuera, en el viejo patio del cementerio, salían de la nieve muchas cruces. Aquel lugar aún tenía la tristeza de la muerte romántica. Pero muy pronto los muertos de Moscú, elevados en sus pequeñas copas, decorarán los jardines donde jueguen los niños y vivirán entre los troncos de los árboles, junto a los nidos de los pájaros, alegremente. (Alberti 1969: 351)

El poeta gaditano ha explicado el proceso de la cremación y su practicidad, así como su reciente generalización en la URSS como sustitución del entierro. La reducción de la muerte a un proceso biológico y a una transformación material, lejos de un sentido religioso que produce "tristeza", permite que la convivencia de los hombres con los restos de la gente que ya ha muerto sea distinta: los muertos “decorarán” y "vivirán [...] alegremente” (Alberti 1969: 351). Tanto en el caso de Alberti como en el de Vallejo hay un paso a una emocionalidad genuina (el amor sin clases) o positiva (la alegría de la muerte con una mirada atea). ${ }^{9}$ Por lo tanto, ante la mirada de ambos autores las emociones toman otro valor, mudan de sentido o recuperan la autenticidad que la sociedad clasista había alterado.

Una nueva moral (o nuevo moralismo) se constituye en la Unión Soviética entre la década de los veinte y la de los treinta, y ambos escritores se esfuerzan en idealizarla (o idealizarlo) a través de una selección. El examen de Vallejo al respecto es menos blando que el de Alberti (cf. Salaün 2004: 144-145), aunque los dos escriben para periódicos (en clave combativa) y tienen fines persuasivos. No obstante, como ya se ha mencionado a propósito de su posición programática que trata de evitar las emociones y su posterior incursión en un tono que se puede calificar de “emocional”, las crónicas de Vallejo contienen una falta de coherencia entre lo que el autor se propone hacer y lo que al final termina escribiendo. Por otra parte, ambos escritores navegan durante los primeros años de la década de los treinta en medio de un ambiente tenso, que orilla a tomas de partido tajantes. En esa navegación la vida está en juego, como en el homicidio de Federico García Lorca el 18 de agosto de 1936 en Granada. ${ }^{10}$

Al examinar las emociones de una sociedad tan distinta a aquellas donde residen, Vallejo y Alberti dependen de intérpretes que posibiliten la comunicación verbal (con algunos rusos, no obstante, debieron de poder hablar directamente en francés, especialmente con los intelectuales). Dejando a un lado el hecho problemático de las distorsiones lingüísticas, es necesario apuntar que la comunicación a veces depende de la observación de los gestos -según Alberti, él y un escritor "[llegan] casi a arrancar[se] uno a otro los botones de la

9 Hay que señalar que Alberti llega al crematorio en compañía de los "Sin Dios”, "los organizadores de la Liga Antirreligiosa de la Unión Soviética” (Alberti 1969: 351).

10 Alberti señala tener la certeza de que él tenía que ser asesinado en vez del poeta granadino, como relata en un pasaje de La arboleda perdida (cf. Alberti 1987: 269). 
chaqueta para dar expresión a [su] charla” (Alberti 1969: 346) - o de los hábitos de los individuos encontrados (los reportajes de Vallejo siguen a algunos de los personajes hasta las esferas más íntimas de su vida diaria). Además, las observaciones sobre las emociones deben mucho a las nociones generales sobre marxismo-leninismo que ambos escritores han adquirido. Así, a diferencia de otros tipos de viajes, donde el viajero deposita algunos de sus prejuicios sobre la gente del país, siendo los prejuicios diversos y hasta contradictorios entre sí, en el caso del viaje a la URSS el viajero intelectual de izquierdas configura a priori, teóricamente, la naturaleza del Homo sovieticus; de ello depende que muchas de las personas -o de los personajes- encarnen más bien tipos políticos con emociones ideológicamente prefabricadas.

Una indagación en las configuraciones de estos temas permite conocer varias de las líneas a partir de las cuales se desarrolla la poesía de ambos escritores en la década de los treinta. Asimismo, contribuye al estudio de la historia intelectual a partir de las simpatías, empatías y antipatías de las redes de escritores y artistas de izquierda en Europa y América (Vallejo no volvió a Perú), sobre todo teniendo en cuenta la queja casi general de los académicos que incursionan en el giro emocional: que a menudo las emociones han sido "ignoradas" en la tradición intelectual (Weyher 2012: 343) y que, en las lecturas de Marx, se ha dado prioridad a la parte racional de la (falsa) "dicotomía” razón / emoción (Weyher 2012: 347). Por otra parte, pocos géneros dependen tanto y tan explícitamente del arsenal de las emociones como la poesía, en particular la poesía lírica, y Alberti y Vallejo son buenos ejemplos de la relación del género lírico con la militancia política -en términos radicales, tanto en una como en otra labor-; en este sentido, sus observaciones y sus palabras sobre la cuestión de las emociones soviéticas dentro de sus textos prosísticos se pueden cotejar con las diferentes modulaciones de sus voces en el ámbito de la poesía. En el caso de Alberti, hay una correspondencia entre un ámbito y otro. En el de Vallejo, la relación es más complicada y todavía no se ha esclarecido.

Finalmente, una pesquisa en esta línea de investigación permite identificar las modulaciones de la emoción (y la pasión) que dirigen las producciones textuales de ambos escritores (y, en general, de los escritores tanto de América como de Europa en el horizonte exaltado de esa época), para intentar un examen de los modos en que un tipo de desplazamiento específico, con un vector determinado (que se puede dirigir hacia Moscú, la fábrica soviética o el Kremlin, con sus respectivos ciudadanos soviéticos, obreros o Stalin), permite que los autores hayan incidido en sus comunidades de origen a través del tratamiento verbal de las emociones y las pasiones como herramientas de la persuasión (en el sentido aristotélico). Tras moverse, ellos intentan mover a los demás, y así ejecutar un papel revolucionario, según un "vínculo emocional entre pensamiento y ac- 
ción” (como analiza L. Frank Weyher) que está en la base de la teoría marxista de la alienación (Weyher 2012: 356) y, por lo tanto, de la "solidaridad de clase" y de la "lucha de clases" (Weyher 2012: 347). Tanto la "solidaridad de clase" como la "lucha de clases" dirigirán los derroteros de ambos poetas en los años posteriores al viaje $\mathrm{y}$, en varios sentidos, de la historia mundial durante los conflictos bélicos en las siguientes décadas. Evaluar los relatos de viaje en relación con las configuraciones emocionales de la época nos permite una de las tareas que propone Sabine Hake: "treating political emotions as aesthetic phenomena” (Hake 2017: 2), y, en consecuencia, contribuir a la comprensión de estos textos no solo como tomas de partido, sino también como configuraciones literarias dentro de la cultura de izquierdas en el periodo de entreguerras.

\section{Bibliografía}

Ahmed, Sara (2014): The Cultural Politics of Emotion. Edinburgh / New York: Edinburgh University Press / Routledge.

Alberti, Rafael (1969): “Rafael Alberti: un reportage inédit sur son voyage en U.R.S.S.”. En: Bulletin Hispanique 71.1-2, pp. 335-353.

Alberti, Rafael (1970): Prosas encontradas (1924-1942). Ed. Robert Marrast; Pról. Pablo Corbalán. Madrid: Ayuso.

Alberti, Rafael (1976): La arboleda perdida. Barcelona: Seix Barral.

Cabrol, Isabel (2004): "La poésie de Rafael Alberti au tournant des années 20 et 30: Le dépassement des avant-gardes esthétiques par les avant-gardes politiques”. En: Salaün, Serge / Carandell, Zoraida (eds.): Rafael Alberti et les avant-gardes. Paris: Presses Sorbonne Nouvelle, pp. 119-138.

Courtois, Stéphane et al. (1997): Le Livre noir du communisme. Paris: Robert Laffont. García, Miguel Ángel (2017): "La lírica comunista que pudiera venir de Rusia: Rafael Alberti (1930-1939)”. En: Gómez L-Quiñones, Antonio / Winter, Ulrich (eds.): Cruzar la línea roja. Hacia una arqueología del imaginario comunista ibérico (1920-2017). Madrid / Frankfurt a. M.: Iberoamericana / Vervuert, pp. 45-70.

Gómez L-Quiñones, Antonio / Winter, Ulrich (2017): “Introducción: Algunos problemas históricos y teóricos del comunismo ibérico”. En: Gómez L-Quiñones, Antonio / Winter, Ulrich (eds.): Cruzar la línea roja. Hacia una arqueología del imaginario comunista ibérico (1920-2017). Madrid / Frankfurt a. M.: Iberoamericana / Vervuert, pp. 9-43. Hake, Sabine (2017): The Proletarian Dream. Socialism, Culture, and Emotion in Germany, 1863-1933. Berlin / Boston: De Gruyter.

Hollander, Paul (1981): Political Pilgrims. Travels of Western Intellectuals to the Soviet Union, China, and Cuba, 1928-1978. New York: Oxford University Press.

León, María Teresa (1987): Memoria de la melancolía. Pról. Rafael Alberti. Barcelona: Círculo de Lectores. 
Leys Ruth (2011): “The Turn to Affect: A Critique”. En: Critical Inquiry 37.3, pp. 434-472. 〈https://doi.org/10.1086/659353〉.

Marrast, Robert (1986): “Le deuxième voyage de Rafael Alberti en URSS: Nouvelles proses retrouvées”. En: Bulletin Hispanique 88.3-4, pp. 357-384.

Salaün, Serge (2004): “Rafael Alberti et la guerre: une épopée oubliée”. En: Salaün, Serge / Carandell, Zoraida (eds.): Rafael Alberti et les avant-gardes. Paris: Presses Sorbonne Nouvelle, pp. 139-153.

Vallejo, César (2002): Ensayos y reportajes completos. Ed. Manuel Miguel de Priego. Lima: Pontificia Universidad Católica del Perú.

Weyher, L. Frank (2012): “Re-Reading Sociology via the Emotions: Karl Marx's Theory of Human Nature and Estrangement”. En: Sociological Perspectives 55.2, pp. 341-363. <https://doi.org/10.1525/sop.2012.55.2.341>. 
